Nataliia Matvieieva*, Constanze Neupetsch, Markus Oettel, Varun Makdani and WelfGuntram Drossel

\title{
A novel approach for increasing the traceability of 3D printed medical products
}

\begin{abstract}
According to the European Medical Device Directive (MDR (EU) 2017/745), medical products of all risk classes are obliged to be labelled with Unique Device Identification Codes (UDI). In order to meet the requirements of the MDR, a novel method of identification for additively manufactured metallic products was developed at the Fraunhofer Institute for Machine Tools and Forming Technology (IWU). The method provides a product traceability within its production process and during the whole product lifespan, and also functions as a counterfeit protection. This paper shows the integration of a unique identification during an additive manufacturing process for a titanium part. The readout of the integrated identification was executed using ultrasonic and eddy current methods, as well as micro computed tomography. Obtained results are discussed in the paper.
\end{abstract}

Keywords: MDR, Component Identification, Traceability, Barcode, Laser Beam Melting, Powder Bed Fusion

https://doi.org/10.1515/cdbme-2020-3081

\section{Introduction}

Recently, a growing demand for additively manufactured medical products is observed [1, 2]. In particular, metal additive manufacturing (AM) is widely used in branches of industry such as customized prosthesis and orthotics [3-5], smart implants [6, 7], dentistry [8, 9] and medical instrumentation [10], etc., where the number of AM products is continuously rising. Additive manufacturing provides a flexible [11], individual [12], time- and cost-saving [13-14] fabrication, which explains a growing interest of medical industry [15].

\footnotetext{
*Nataliia Matvieieva: Fraunhofer Institute for Machine Tools and Forming Technology IWU, Nöthnitzer Straße 44, Dresden, German, e-mail: Nataliia.Matvieieva@iwu.fraunhofer.de Constanze Neupetsch, Markus Oettel, Varun Makdani, WelfGuntram Drossel: Fraunhofer IWU, Dresden, Germany
}

At the same time, the medical industry of Europe faces some challenges, which are caused due to the identification of medical products in accordance to the MDR. Product tracking during the whole product's lifespan implied by this regulation. In practice, product tracking during the production cycle is achieved by applying various identifiers to the surface of the product, employing, for instance, laser labelling or stickers. However, the identifier is absent during the process of sortation, following the product manufacturing, which might cause mistakes and fails the requirements of product tracking. Currently, the realization of tracking a specific product (e.g. implant) is not always possible due to the deficient access to patient-specific implant data and the absence of an appropriate identification method with a non-invasive in vivo verification. In order to address these issues (problems), a novel method for metal AM product identification was developed. Based on it, the product's tracking could be done right after its design and during the whole lifespan (even in vivo). Moreover, the type of identifier provides also a counterfeit protection, which can be useful due to the growing AM market.

\section{Methodology}

The aim of the current work is to develop and demonstrate the tracking method for the AM product during its production and life cycle. The chosen identifier will be manufactured inside the component during its production, then read-out and decoded, employing common non-destructive methods such as eddy current, ultrasonic techniques and computed tomography.

The proposed identification technique for additive manufacturing methods can be divided in three stages. The first stage is the CAD design of the product with an integrated identifier and the manufacturing process (Fig.1 a). The second stage involves the parameters' adjustment of the chosen nondestructive methods to the embedded identifier's type, dimensions and position, with its subsequent read-out (Fig. 1 b). At the third stage, obtained signals are processed and decoded (Fig.1 c). 


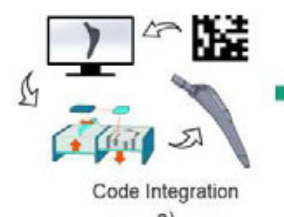

a)

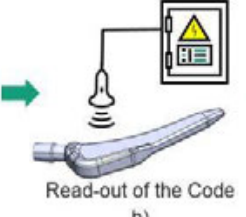

b)

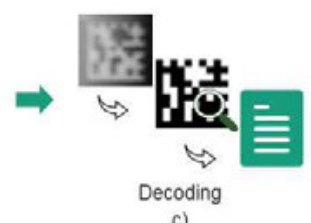

c)
Figure 1. Proposed identification method of AM products

\section{Experiment}

\subsection{Specimens}

Laser Beam Melting (LBM) or also called laser powder bed fusion is an additive manufacturing technology. Therein, metal powders are used as base material for generating a defined solid three-dimensional structure instead of subtracting or deforming material in conventional manufacturing like cutting, turning or forming. LBM is a layer based manufacturing process. The input $3 \mathrm{D}-\mathrm{CAD}$ models have to be sliced in layers between 20 and $150 \mu \mathrm{m}$ thickness. The metal powder is applied out of a container (powder stock) for each layer and in between selectively molten by a laser according the part geometry (cross section). The molten powder solidifies rapidly to a solid body with a density above $99 \%$ according to VDI 3405, Part 2 [16].

The test specimen with the integrated unique identifier (barcode) were manufactured from Titanium (Ti6Al4V) using $25 \mu \mathrm{m}$ thin layers on a Concept Laser M2 Cusing machine ( $400 \mathrm{~W}$ single laser). A scan speed up to $1500 \mathrm{~mm} / \mathrm{s}$ and a laser power up to $370 \mathrm{~W}$ were used for the LBM process.

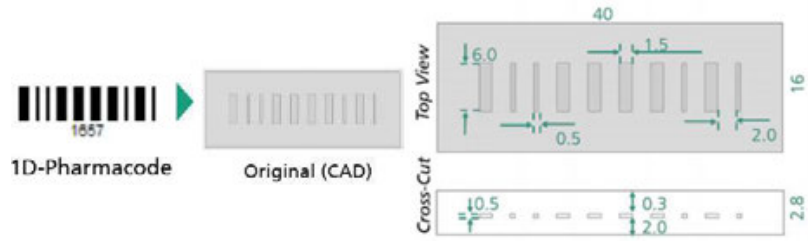

Figure 2. Titanium specimen with integrated barcode and its $C A D$ design (left) and dimensions (right)

The bars of the code insight the specimen are cavities with non-molten metal powder inside. On Fig. 2 (left) the barcode (type pharmacode, the coded number is ' 1657 ') and its CAD image are presented. The dimensions of the specimen are $40 \times 16 \times 2.8 \mathrm{~mm}^{3}$, where the identifiers' size is $30.8 \times 6 \times 0.5$ $\mathrm{mm}^{3}$. The code consists of two bar elements, with 1.5 and 0.5 $\mathrm{mm}$ widths, grouped on $2 \mathrm{~mm}$ distance from each other (Fig. 2 right). The depth on which the barcode was integrated is 0.3 $\mathrm{mm}$ from the top and $2 \mathrm{~mm}$ from the bottom side.

In these works, three non-destructive methods were used to read-out hidden identifier and receive coded data.

\subsection{Eddy Current Method}

\subsubsection{Barcode detection}

The first method is the eddy current (EC) measuring technology, which is generally used for the quality assurance of conductive materials. The method is sensitive to the smallest cracks in metal, but limited by the sensor's operational frequency. The higher the frequency the better the resolution and sensitivity to small structures/defects. But at the same time the penetration depth in the investigated specimen is exponentially decreasing in accordance to the skin layer law, which makes it very difficult to obtain signals from deeplaying identifiers. In the experiment, the eddy current scanning device MPECS EddyCus was used to scan the code employing a cylindrical absolute coil (outer diameter - $2.5 \mathrm{~mm}$, inner diameter (ferrite) - $1 \mathrm{~mm}$ and resonant frequency is $7.5 \mathrm{MHz}$ ) at frequency of $1 \mathrm{MHz}$ and lift-off of $0.1 \mathrm{~mm}$. The coil has a ferrite outer shield, combined with a relatively small diameter, which results in a quite good resolution of the measuring spot. The measurement was performed line by line with OY and OX spatial resolution of $0.2 \mathrm{~mm}$ per pixel. The scan of the integrated identifier is demonstrated in Fig.3, EC (a) and the signal values are represented as a $2 \mathrm{D}$ grey-scale image, where the white regions correspond to the decreased conductivity in the specimen (bars). As it is seen from the image, the scanned barcode repeats the original one, the number of bars, their width and order are clearly recognizable.

\subsubsection{Signal processing and data decoding}

To extract the coded data from the identifier, the signal processing and decoding algorithm was done in Python. In the scope of the current work, image denoising was performed using Gaussian filter and contrast enhancing was carried out with the help of histogram equalization. The region on the scan (Fig. 3, EC b), marked with red rectangle, was analyzed. The profiling graph of the extracted region was built and is presented in Fig. 3, EC c, where the analyzed signal is the averaging intensity of the sum of horizontal scans. On the profiling graph (total signal), the extremums of peaks and their mean widths were found. For a signal decoding, the parameters of each peak were evaluated as a $k=\sqrt{a^{2}+b^{2}}$, where $k$ is an analyzed parameter, $a$ is a prominence of the peak and $b$ is a mean width of a peak. After setting appropriate thresholds of parameter $k$ for " 0 " (small thin peaks) and for " 1 " (high wide peaks) the signal was correctly decoded (1 00 $\left.\begin{array}{lllllll}1 & 1 & 1 & 1 & 0 & 1 & 0\end{array}\right)$. The algorithm was used for 10 different 
measurements of the same barcode, each measurement was done only for a rectangle region (Fig. $3 \mathrm{EC} \mathrm{b}, 6$ scanned lines for 14 seconds). For all the measurements correct results of decoding were achieved.

\subsection{Ultrasonic Method}

\subsubsection{Barcode detection}

The second chosen method was ultrasonic testing (US), which is based on the propagation of ultrasonic waves into the tested material. Based on the obtained reflections, the material's properties are characterized and quality is assured. As well as for eddy current technique, the inspection resolution depends on the frequency at which the material is investigated. The better resolution is obtained for higher frequency, however the signal attenuation rate is also higher.

The 1D linear array ultrasonic transducer was used for the 1D barcode detection. The array consists of 64 elements $(0.5 \mathrm{~mm}$ raster) and has the active square $32 \times 7 \mathrm{~mm}^{2}$.

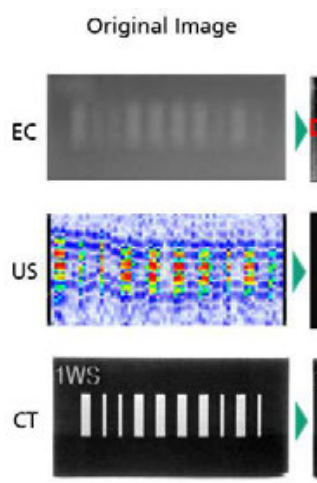

a)
Processed Image

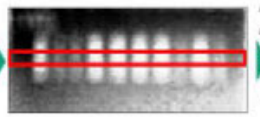

Inthin:
Signal Intensity over coordinate
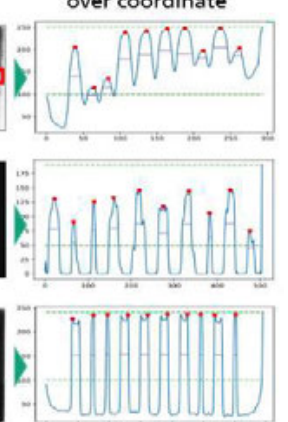

Figure 3. Results for the identifier decoding, a) original image; b) processed image; c) transformed and decoded image

The working frequency, at which the desirable resolution was received and barcode clearly detected, was $10 \mathrm{MHz}$. The US scanning was performed with a distance of $6.4 \mathrm{~mm}$ from the surface in water coupling with a focus distance of $2 \mathrm{~mm}$. However, it is also possible to use such types of arrays also with a soft-rubber pad coupling, which makes it possible to employ such US technique in the production line. The measurement results were acquired in real time and represented as a 2D RGB image, where red regions correspond to the higher amplitude of the reflected US signal from the integrated bar. The pixels resolution $(0.5 \mathrm{~mm})$ had the same dimension as the piezo element width. The received image is presented in the Fig. 3, US a.

\subsubsection{Signal processing and data decoding}

The US signal processing and decoding are based on the same algorithm as the developed method for the eddy current technique. After receiving the 2D RGB image, it was converted to the grey-scale image, denoised (separating the informative signal from the background) and the contrast was enhanced (Fig. 3, US b). Hereafter, the total mean pixel values (signal amplitudes) for each bar were calculated and built-up on the profiling graph (Fig. 3, US c). Based on the prominence and width of the peak, the decoding was performed. The experiment was conducted 5 times for the same barcode, where some signal variations were observed. However, that have not influenced the results of the decoding.

\subsection{Computed Tomography Method}

\subsubsection{Barcode detection}

The third chosen method is the X-ray imaging method, which due to its high preciseness and accuracy allows detecting defects in material in micron range. The method is based on employing the X-ray irradiation for receiving a 2D structural data of the object.

In the research, the vItomeIx s 240 (GE Sensing \& Inspection Technologies GmbH, Germany) computed tomography (CT) device was employed for receiving $2 \mathrm{D}$ images of the specimen (Fig. 3, CT a).

\subsubsection{Signal processing and data decoding}

The received CT image of the chosen specimen was very precise and with high contrast, therefore the data decoding could be done without extra image processing. However, in a purpose of the method universalisation, the same processing algorithm was done for CT image as well. In the Fig. 3, CT b, the denoised image with increased contrast is presented. The next step of successful data decoding, in analogue EC and US, was done and is presented in the Fig. 3, CT c.

\section{Discussion}

The evaluation of the received signals (images) applying different non-destructive methods has shown that the determinative factor in signal analysis for EC technique is the change of the signal amplitude in case of different structure 
(bar) width. Which is in a contrast to the US and CT, where the width of the signal peak closely corresponds to the width of the structure (bar). That could be explained by the physics of the EC signal formation, where measuring the broad bar (formed as void), the signal has higher amplitude due to the lower density of the EC in comparison to the narrow bars, where the density of the eddy currents is almost the same as in solid material (Fig. 3, EC b). Moreover, the bar's edges are blurred and the real dimensions are distorted due to the specifics of the electromagnetic field propagation that depends on the operating frequency and size of the sensor. To increase the resolution it is possible to employ the other type of the coil (e.g. square), type of sensor (e.g. half-transmission) or changing its dimensions.

The analysis of the US images is mostly based on the measured width evaluation, where the broader the width, the broader the reflective surface for US waves. The enhancement of the resolution is possible in two ways: adjusting the US software (where signal delays in array are analyzed) or using the US transducers with narrower element width.

The CT image has the highest resolution and contrast in comparison to EC and US methods and the data decoding can be performed based on the bars' widths evaluation. However, after integration of the identifier into the component (e.g. implant), which is planned to be tracked in vivo, using conventional X-ray method, the contrast, as well as resolution could decrease significantly. Therefore, relevant experiments have to be performed and sustainable CT image processing algorithm has to be developed.

\section{Conclusion}

In current paper results of the proposed method for additively manufactured medical products identification are presented and discussed. Based on the idea of the identifier integration inside the component in the production process and its readout, successful product tracking is possible to execute right after the product's production (avoiding separate step of the identifier application) as well as to provide a counterfeit protection. For such products' group as implants, the method allows also in vivo tracking, which was proved within the experiment with the help of micro-CT.

For future work it is planned to improve further methods, where the possibilities and techniques for the resolution enhancement of US and EC testing methods will be studied.

\section{Author Statement}

Research funding: The authors have no conflict of interest in relation to the present study. The conducted research is not related to either human or animals use. The work presented here will be co-financed by the Fraunhofer-Gesellschaft.

\section{References}

[1] Javaid M., Haleem A., Additive manufacturing applications in medical cases: A literature based Review, Alexandria Journal of Medicine, 2018; 54: 411-422.

[2]. Wong KV, Hernandez A. A review of additive manufacturing. ISRN Mech Eng.2012; 1-10.

[3]. Noorani R. Rapid prototyping: principles and applications. Hoboken (NJ): John Wiley and Sons Inc; 2006. ISBN:978-0471-73001-9.

[4]. Balazic M, Kopac J. Improvements of medical implants based on modern materials and new technologies. J Achieve Mater Manuf Eng. 2007;25:31-34.

[5]. Milovanovic J, Trajanovic M. Medical applications of rapid prototyping. Mech Eng. 2007;5:79-85.

[6]. Töppel T, et al. Structural integration of sensors/actuators by laser beam melting for tailored smart components. The journal of the Minerals. Metals \& Materials Society 2018;70 (3):321327

[7]. Rotsch C, et al. Concept of an implant with an integrated sensor actuator system for the monitoring and influencing of the mechanical implant bone interface. Biomedizinische Technik 2017;62 (1): 64-66

[8]. Dawood, A., Marti, B., Sauret-Jackson, V. et al. 3D printing in dentistry. Br Dent J 2015;219:521-529.

[9]. Bhargav A., et al. Applications of additive manufacturing in dentistry: A review, Journal of Biomedical Material research Part B, 2018;106 (5):2058-2064.

[10]. Van Noort R. The future of dental devices is digital. Dent Mater. 2012;28:3-12.

[11]. Dahake SW, et al. Applications of medical rapid prototyping assisted customized surgical guides in complex surgeries. Rapid Prototyp J. 2016;22:934-946.

[12]. Yaxiong L, et al. The customised mandible substitute based on rapid prototyping. Rapid Prototyp J. 2003;9: 67-174.

[13]. Kochan A. Rapid prototyping gains speed, volume and precision. Assembly Automation. 2000;20:295-299.

[14]. Evila LM, et al. Rapid tooling using 3D printing system for manufacturing of customized tracheal stent. Rapid Prototyp J. 2014;20:2-12.

[15]. Neupetsch C, et al. Development and Validation of Bone Models using Structural Dynamic Measurement Methods. Current Directions in Biomedical Engineering 2019;5(1):343346

[16]. Additive Fertigungsverfahren, Rapid Manufacturing Strahlschmelzen metallischer Bauteile Qualifizierung, Qualitätssicherung und Nachbearbeitung, 2012;VDI 3405, Blatt 2 